\title{
Time to harness the pro-apoptotic property of $\mathrm{NF} \kappa \mathrm{B}$ ?
}

\author{
Radhakrishnan, S. K. and Kamalakaran, S. \\ e-mail: senthil@uic.edu
}

We read with great interest the review article by Nakanishi and $\mathrm{Toi}^{1}$, in which the authors highlight the usefulness of nuclear factor- $\kappa \mathrm{B}(\mathrm{NF \kappa B})$ inhibitors in anticancer therapy. This is in line with the central theme of NFKB biology, which portrays NFKB as oncogenic and antiapoptotic. However, as the authors themselves briefly mention towards the end of the article, evidence is accumulating in support of a surprising pro-apoptotic role for NFKB. Arguably, the most striking demonstration of NFKB's involvement in cell death first came 5 years ago, when it was reported that NFKB is necessary for p53-mediated apoptosis ${ }^{2}$. So, in situations in which p53 activation in response to chemotherapy is the main mode of elimination of tumour cells, inhibition of $\mathrm{NFKB}$ might well be counterproductive. More recently, it was found that activation of NFKB is essential for doxorubicin and its analogues to mediate their cytotoxic effects, further underscoring the direct death-promoting role of $\mathrm{NF \kappa B}^{3}$. These data do not, by any means, invalidate the earlier studies that characterized $\mathrm{NF \kappa B}$ as anti-apoptotic. Rather, these observations emphasize that there is another side to $\mathrm{NF \kappa B}$ that deserves equal attention.

It is now clear that several factors, including the cell-type and stimulus used, act together to confer a pro-apoptotic role for NFKB in certain situations. ${ }^{4}$. There seems to be two major mechanisms through which NFKB induces apoptosis. The first is by transcriptional upregulation of pro-apoptotic target genes such as TP53, death receptor 4 (DR4), DR5 and tumour-necrosis factor (TNF)-related apoptosis-inducing ligand $(T R A I L)^{5-8}$. The second is by active repression of anti-apoptotic target genes such as $B C L X_{L}$ and XIAP (X-linked inhibitor of apoptosis) ${ }^{9}$. It should be pointed out that these two mechanisms are not mutually exclusive and can occur together ${ }^{10}$.

Despite a considerable amount of work being undertaken in this area in recent years, it still remains to be elucidated why NFKB that is induced by the classic TNF-signalling pathway normally induces a cell survival pathway, whereas NF- $\mathrm{KB}$ activation that is induced by certain other stimuli leads to cell death. It has been suggested that differences in the post-translational modification of the NFKB subunits, especially p65/RELA, could cause a differential response ${ }^{11}$. Also, the subset of genes that are induced by NFKB could be influenced by the status of the chromatin in specific target genes (especially histone-acetylation status) and whether access to these genes is altered in response to particular stimuli. These factors could tilt the balance towards life or death ${ }^{12}$.

We hope that a better understanding of the events that actually shape the NFKB response will help foster new ways for superior anticancer therapies to be developed. For example, instead of screening for inhibitors of NFKB, we might be able to come up with strategies that switch NFKB from being anti-apoptotic to pro-apoptotic.

Senthil K. Radhakrishnan* \& Sitharthan Kamalakaran ${ }^{\ddagger}$

*Departments of Medicine, Microbiology and Immunology,

${ }^{\ddagger}$ Department of Biochemistry and Molecular Biology, University of Illinois at Chicago, Chicago, Illinois, USA.

"Present Address:

Cold Spring Harbor Laboratory, Cold Spring Harbor, New York, USA. 
1. Nakanishi, C. \& Toi, M. Nuclear factor-kB inhibitors as sensitizers to anticancer drugs. Nature Rev. Cancer 5, 297-309 (2005).

2. Ryan, K. M., Ernst, M. K., Rice, N. R. \& Vousden, K. H. Role of NF-кB in p53mediated programmed cell death. Nature 404, 892-897 (2000).

3. Ashikawa, K., Shishodia, S., Fokt, I., Priebe, W. \& Aggarwal, B. B. Evidence that activation of nuclear factor- $\mathrm{\kappa B}$ is essential for the cytotoxic effects of doxorubicin and its analogues. Biochem. Pharmacol. 67, 353-364 (2004).

4. Kucharczak, J., Simmons, M. J., Fan, Y. \& Gelinas, C. To be, or not to be: NF-кB is the answer - role of Rel/NF-KB in the regulation of apoptosis. Oncogene 22, 89618982 (2003).

5. Fujioka, S. et al. Stabilization of p53 Is a novel mechanism for proapoptotic function of NF-кB. J. Biol. Chem. 279, 27549-27559 (2004).

6. Shetty, S. et al. Transcription factor NF-кB differentially regulates death receptor 5 expression involving histone deacetylase 1. Mol. Cell. Biol. 25, 5404-5146 (2005).

7. Jin, F. et al. Activation of nuclear factor- $\kappa B$ contributes to induction of death receptors and apoptosis by the synthetic retinoid CD437 in DU145 human prostate cancer cells. Cancer Res. 65, 6354-6363 (2005).

8. Rivera-Walsh, I., Waterfield, M., Xiao, G., Fong, A. \& Sun, S. C. NF-кB signaling pathway governs TRAIL gene expression and human T-cell leukemia virus-I taxinduced T-cell death. J. Biol. Chem. 276, 40385-40388 (2001).

9. Campbell, K. J., Rocha, S. \& Perkins, N. D. Active repression of antiapoptotic gene expression by RelA ${ }^{\mathrm{p} 65} \mathrm{NF}-\kappa \mathrm{B}$. Mol. Cell 13, 853-865 (2004).

10. Farhana, L., Dawson, M. I. \& Fontana, J. A. Apoptosis induction by a novel retinoid-related molecule requires nuclear factor- $\kappa B$ activation. Cancer Res. 65, 49094917 (2005).

11. Miyamoto, S. RelA life and death decisions. Mol. Cell 13, 763-764 (2004).

12. Graham, B. \& Gibson, S. B. The two faces of NFKB in cell survival responses. Cell Cycle 4,1342-1345 (2005). 